\title{
Evaluating the robustness of a contact-less mHealth solution for personal and remote monitoring of blood oxygen saturation
}

\author{
Gabriella Casalino $^{1} \cdot$ Giovanna Castellano $^{1} \cdot$ Gianluca Zaza $^{1}$ D \\ Received: 12 February 2021 / Accepted: 1 December 2021 \\ (c) The Author(s), under exclusive licence to Springer-Verlag GmbH Germany, part of Springer Nature 2021
}

\begin{abstract}
MHealth technologies play a fundamental role in epidemiological situations such as the ongoing outbreak of COVID-19 because they allow people to self-monitor their health status (e.g. vital parameters) at any time and place, without necessarily having to physically go to a medical clinic. Among vital parameters, special care should be given to monitor blood oxygen saturation $\left(\mathrm{SpO}_{2}\right)$, whose abnormal values are a warning sign for potential COVID-19 infection. $\mathrm{SpO}_{2}$ is commonly measured through the pulse oximeter that requires skin contact and hence could be a potential way of spreading contagious infections. To overcome this problem, we have recently developed a contact-less mHealth solution that can measure blood oxygen saturation without any contact device but simply processing short facial videos acquired by any common mobile device equipped with a camera. Facial video frames are processed in real-time to extract the remote photoplethysmographic signal useful to estimate the $\mathrm{SpO}_{2}$ value. Such a solution promises to be an easy-to-use tool for both personal and remote monitoring of $\mathrm{SpO}_{2}$. However, the use of mobile devices in daily situations holds some challenges in comparison to the controlled laboratory scenarios. One main issue is the frequent change of perspective viewpoint due to head movements, which makes it more difficult to identify the face and measure $\mathrm{SpO}_{2}$. The focus of this work is to assess the robustness of our mHealth solution to head movements. To this aim, we carry out a pilot study on the benchmark PURE dataset that takes into account different head movements during the measurement. Experimental results show that the $\mathrm{SpO}_{2}$ values obtained by our solution are not only reliable, since they are comparable with those obtained with a pulse oximeter, but are also insensitive to head motion, thus allowing a natural interaction with the mobile acquisition device.
\end{abstract}

Keywords mHealth $\cdot$ Self-monitoring $\cdot$ COVID-19 $\cdot$ Blood oxygen saturation $\cdot$ Signal and Video Processing $\cdot$ Remote Photopletismography - rPPG

\section{Introduction}

The current COVID-19 (Coronavirus Disease 2019) pandemic has changed our daily lives, by imposing some strict behavioural uses, such as wearing mask, distancing, and in most severe conditions lockdown. At the time of writing this paper, many countries worldwide are still trying to limit the spread of the virus by requiring people to remain

Gianluca Zaza

gianluca.zaza@uniba.it

Gabriella Casalino

gabriella.casalino@uniba.it

Giovanna Castellano

giovanna.castellano@uniba.it

1 Department of Computer Science, University of Bari "Aldo Moro", Bari, Italy in self-isolation at home and limit human contacts as much as possible. On the other side, hospital facilities have been taken by storm by the huge inflow of Covid patients, also causing slowdowns in treatment of other diseases. Despite the arrival of the first vaccines against Corona virus, these precautionary measures will have to be observed for a long time to come.

In this context, mHealth, which is a new frontier of telehealth encompassing the use of mobile and wireless technologies to support digital health services, assumes crucial importance (Lupton 2013; Swan 2012; Ardito et al. 2020; Tripathi et al. 2020). Thanks to mHealth solutions, that allow people to self-monitor their vital parameters in any place and at any time through the use of mobile devices, patients can have their vital signs continuously monitored without having to physically go to a medical clinic, thus reducing the risk of virus transmission (Kim and Choi 2020). Moreover, 
the monitoring of vital signs undertaken by mobile devices allow immediate medical intervention when there is a deterioration in the health status, and reduces unnecessary hospital admissions, time and costs spent on transport, and healthrelated costs. Actually, the potential for using mHealth and in general telehealth solutions during emergencies has been emphasized in Lurie and Carr (2018) and even WHO (World Health Organization) has given specific highlight of telehealth as a powerful tool to curb the spread of the Corona virus and for early detection of the disease at the very onset. In particular, monitoring blood oxygen saturation $\left(\mathrm{SpO}_{2}\right)$ is of fundamental importance for early detection of COVID-19 since abnormal values of this vital parameter are a warning sign for potential infection even before patients start having short breath. Indeed, it has been observed ${ }^{1}$. that the $\mathrm{SpO}_{2}$ values may fall below $90 \%$ much more before the first symptoms of Covid pneumonia appear. $\mathrm{SpO}_{2}$ monitoring can be easily done at home through the pulse oximeter that however requires skin contact and hence could be a potential way of spreading contagious infections.

To overcome this problem, in Casalino et al. (2020) we have recently developed a mHealth solution for contact-less estimation of blood oxygen saturation. Its basic idea is based on extracting the photoplethysmographic signal from facial video frames, hence it does not require any skin contact for measurement of the vital sign. The first experimental evidences reported in Casalino et al. (2020) have shown that our mHealth solution works well in controlled environments and provides several advantages. It is cheap, since it just requires a mobile device equipped with a camera (smartphone, laptop, tablet, ...), and does not need specific medical devices. It is contact-less, thus avoiding potential infections. It is easyto-use, since the patient experiences the self-monitoring of vital parameters through a simple and natural action that can be part of his daily routine. Moreover, it can be easily integrated within a patient-centered telehealth platform for both personal and remote health monitoring. Finally, it is fast, since it provides reliable $\mathrm{SpO}_{2}$ measurements in a couple of seconds. Although the proposed solution has been calibrated to perform the measurement of blood oxygen saturation aligned with a common pulse oximeter, there is still a lack of validation studies concerning its applications in real world scenarios. Indeed, measuring the $\mathrm{SpO}_{2}$ using mobile devices in real-world daily situations holds some challenges in comparison to the controlled laboratory scenarios where the first experiments of our solution were conducted. One main issue is the frequent change of perspective viewpoint due to head movements, which leads to challenges in detecting the human's face and computing an estimate of $\mathrm{SpO}_{2}$.

\footnotetext{
1 https://www.nytimes.com/2020/04/20/opinion/sunday/coronavirustesting-pneumonia.html
}

Hence, to better assess the validity of our solution for in-thewild scenarios, it is fundamental to assess its robustness to head movements. This is the goal of the present work. To understand to what extent our mHealth solution is robust to head movements, in this paper we carry out a pilot study on the PURE benchmark dataset that takes into account different head movements during the $\mathrm{SpO}_{2}$ measurement. Experimental results have shown that measurements obtained by our contact-less solution are reliable since they are comparable with those obtained using a commercial pulse oximeter, and are insensitive to head movements.

The paper is organized as follows. In Sect. 2 we overview some related works.

Section 3 describes the problem of $\mathrm{SpO}_{2}$ measurement via photopletismography. Section 4 summarises the proposed solution. In Sect. 5 we discuss experimental results on a benchmark dataset to evaluate how head motion affects the $\mathrm{SpO}_{2}$ measurements. Conclusions and future works are outlined in Sect. 6.

\section{Related works}

Several mHealth solutions have been recently proposed that allow patients to self-monitor their health status and stay remotely in touch with physicians, thus avoiding them to go physically to clinical centers. Most of these solutions rely on the well established technology known as remote photopletismography (rPPG) that has been largely used to develop contact-less solutions for monitoring vital parameters including $\mathrm{SpO}_{2}$. In the pioneering work by Verkruysse et al. (2008), a digital camera in ambient light is used to estimate heart rate and breath rate values based on rPPG. Poh et al. (2010) proposed the use of the blind source separation of colour channels into independent components to process facial videos for heart rate estimation. Hassan et al. (2017) and Rouast et al. (2018) present an overview of rPPG techniques based on images of faces for heart rate estimation. Since vital parameters must be correctly estimated by means of trustable devices, several works studied the limits of these approaches, due to their sensitivity to external factors, such as light in Yang et al. (2021) and Song et al. (2021), variations in environmental conditions and subject demographics in Dasari et al. (2021), presence of face masks in Speth et al. (2021), and motion in Liu et al. (2021). However, none of these works studied the influence of those factors on the measurement of blood oxygen saturation. Only Allado et al. (2021) have recently designed a clinical trial to evaluate the effectiveness of rPPG-based measurements of blood oxygen saturation (as well as heart rate and respiratory rate) compared to those obtained with contact devices.

First attempts to use PPG-based measurements employed specialized and expensive devices that are difficult to retrieve 
and use, thus largely excluding a portion of the potential target users. More recently, low cost devices or common devices such as smartphones have been employed for largescale adoption in daily life. Many free or low-cost software applications specifically designed for self-measuring of vital parameters through smartphones have been recently developed. For example Tayfur and Afacan (2019) use built-in sensor and light source of a Samsung Galaxy S8 smartphone to measure heart rate and $\mathrm{SpO}_{2}$. Chan et al. (2019) evaluates the reliability of a smartphone oximeter in measuring heart rate and $\mathrm{SpO}_{2}$ during rehabilitation therapies. Tomlinson et al. (2018) developed a camera-based application and probe-based application using Apple iPhone 5 and iPhone 6 to measure heart rate and $\mathrm{SpO}_{2}$ of healthy children and the triage values of pediatric patients. In Sahoo et al. (2017), a mobile app is used to track patients' heart activities and to send a warning if anomalous values are detected. In Storz et al. (2021) a mobile app has been designed to support individual monitoring of vital parameters of outpatients.

Further examples on the use of mobile applications for chronic disease monitoring have been systematically reviewed in Moses et al. (2021) and El-Rashidy et al. (2021). Mhealth solutions are widely used for monitoring patients affected by different pathological conditions, such as patients undergoing haemodialysis treatment in Villarroel et al. (2020), patients under home oxygen therapy in Naranjo-Rojas et al. (2021), patients affected by bipolar disorder in Casalino et al. (2021). Currently, mobile solutions have been shown to be also very useful during COVID-19 pandemic. A wide overview, discussing the use of mobile apps for Covid-19 is provided by Kondylakis et al. (2020). As an example El-Rashidy et al. (2020) propose a mobile GPS-based app is used for monitoring patients which are infected or suspected of COVID-19.

Besides smartphones, other cheap and ergonomic mobile devices have been proposed for personal and remote measurement of vital parameters. An example is the smart bracelet developed within the Telemachus (Telemedicine Monitoring and Collaborative Hub-and-Spoke System) project ${ }^{2}$. that allows to remotely monitor Covid patients in all phases of the disease by detecting the vital parameters of the wearer and communicating via geolocalization or via Bluetooth. Another device for data collection and remote monitoring of COVID-19 patients in home isolation is described in the cosinuss ${ }^{\circ}$ project $^{3}$. It consists of a device in the wearable in-ear using photoplethysmography to monitor vital parameters, a data gateway and a cloud database server (Baldinger et al. 2021). In Sabbadini et al. (2021) heart rate and breathing

\footnotetext{
$2 \mathrm{https} / / /$ business.esa.int/projects/telemachus

${ }^{3}$ https://www.cosinuss.com/en/portfolio-items/study-on-remote-patie nt-monitoring-for-covid-19/
}

rate are measured through the use of a face mask using the photoplethysmography technique to detect the presence of environmental and physical stress factors in workers.

Despite the current proliferation of personal mobile/ wearable devices for vital parameter self-monitoring, the state-of-art literature shows that the most suitable sensor for contact-less measurement of $\mathrm{SpO}_{2}$ is the video camera that satisfies both easy-of-use and contact-less requirements. The present work represents an advancement of the state of the art by introducing a general camera-based mhealth solution for the reliable measurement of blood oxygen saturation. Given the absence of studies on the influence of external factors on rPPG measurement of blood oxygen saturation, in this study we investigate the influence of head movements to better assess the robustness of our rPPG-based solution.

\section{Problem definition}

The problem we face in this work is to estimate the value of blood oxygen saturation through a contact-less solution exploiting remote photopletismography. According to Challoner (1979), the photoplethysmography (PPG) signal represents the variation of lights due to cardiovascular activities in some regions of the face tissue. These variations can be used to estimate the hearth rate and the blood oxygen saturation (Allen 2007). Specifically, according to Kong et al. (2013), an estimation of $\mathrm{SpO}_{2}$ can be obtained by considering the cardiovascular pulse wave signal at two different wavelengths, namely the red and blue bands, being the blue band representative of the infrared wavelength used in the traditional pulse oximeter.

The underlying principle is that the impulse of cardiovascular wave that flows through the body periodically, causes stretch in the vessel walls, with consequent fluctuations in blood volume. These fluctuations modulate the absorbency of light passing through a given volume of tissue, so it is possible to evaluate the variation of light during a normal cardiac cycle. There are two methods to implement PPG measurement of $\mathrm{SpO}_{2}$. The first method is contact PPG, in which the user places his/her finger on the sensor and the flash light provides the light source required for the blood cells to become visible (Kim et al. 1986). The second method is contact-less or remote PPG, in which the camera is held in front of the user's face and the measurement is undertaken without the need for skin contact. For the motivations described in section I, we chose to implement a contact-less PPG estimation of $\mathrm{SpO}_{2}$. The following sections outlines the methodological pipeline underlying out contact-less solution. 
Fig. 1 Methodological pipeline for contact-less $\mathrm{SpO}_{2}$ measurement

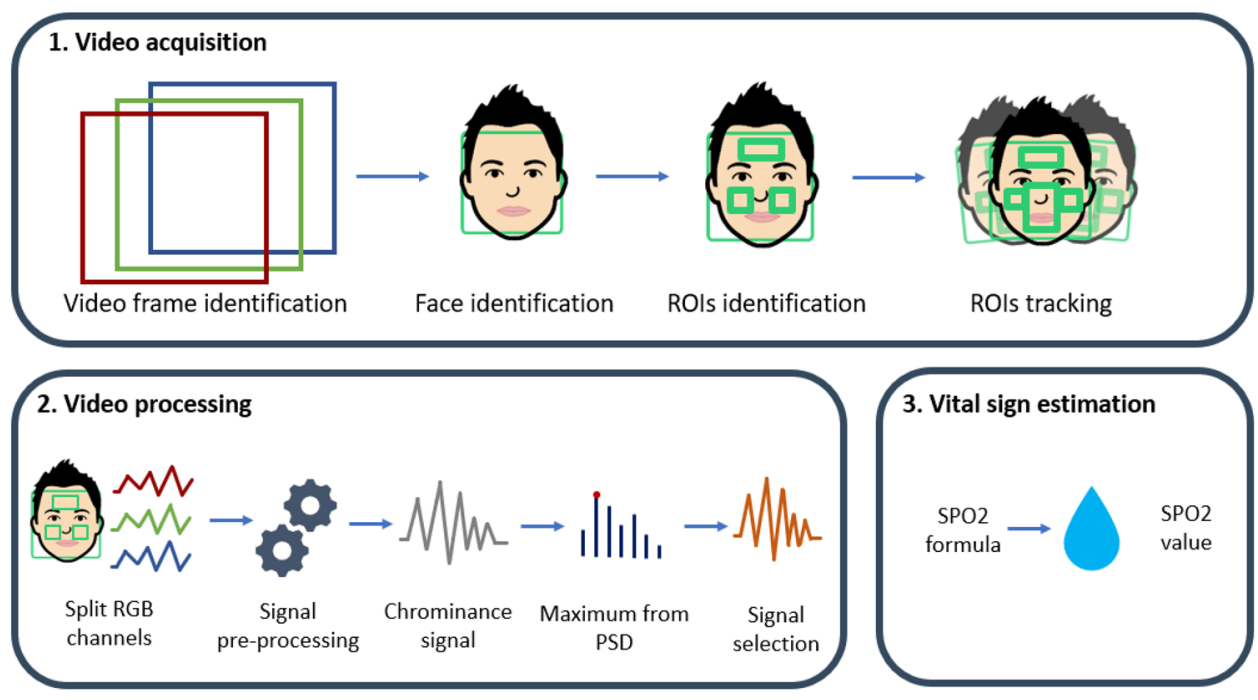

\section{The proposed mHealth solution}

Our approach for contact-less measurement of blood oxygen saturation is based on processing a short video frame sequence that capture the face of the user in order to extract the photopletismographic signal and derive an estimate of $\mathrm{SpO}_{2}$. The detailed description of the methods underlying our solution are described in Casalino et al. (2020). Here we briefly describe the main phases of the methodological pipeline (Fig. 1).

In the first phase, a short video capturing the user's face is acquired through the video camera and a sequence of video frames 2 seconds long is processed. Resolution of the video frames depend on the characteristics of the video camera integrated in the mobile device. Each frame is processed for face detection and three regions of interest (ROIs) are identified, specifically the two cheeks and the forehead, which represent the areas where the plethysmographic signal is most evident (Challoner 1979). We used three different regions in order to make the acquisition more robust to occlusion, such as glasses, beard and so on, as it has been proven in our previous work by Casalino et al. (2020). To cope with head movements, a fourth ROI is located in the middle of the face and used for face tracking. This further ROI is connected with the other three ROIs (cheeks and forehead), so that together they can indicate the movement of the face. This enables face tracking that makes the system less sensitive to movements and thus avoids the user having to stand still in front of the camera.

The second phase is devoted to extract the photopletismographic signal from the identified ROIs. Signal processing techniques are applied to the three different ROIs for noise reduction, and the most informative ROI is selected for $\mathrm{SpO}_{2}$ measurement, as suggested in Solomon (1991). Particularly, for each of the three ROI, the RGB signals are extracted.
Then for each of the three channels (red, blue and green) and for each frame in the video, the average value over all the pixels is computed. Thus a three dimensional signal is derived for each frame. High-frequency noise in signals are filtered (Speake and Mersereau (1981)) and the the chrominance method (De Haan and Jeanne (2013)) is used to limit the influence of motion and illumination variations on the signal. Finally linear interpolation is applied to uniform the signal.

After signal cleaning, red and blue components of the signal are used to compute $\mathrm{SpO}_{2}$ value according to the following formula that was suggested by Kong et al. (2013):

$S p O_{2}=A-B \frac{A C_{R E D} / D C_{R E D}}{A C_{B L U E} / D C_{B L U E}}$

where the $A C_{R E D}$ and $A C_{B L U E}, D C_{R E D}$ and $D C_{B L U E}$, are respectively the standard deviations and the mean of the red and blue signals computed from the sequence frames. $A$ and $B$ are calibration parameters. We used $A=125$ and $B=26$ as in Kong et al. (2013).

Using the above described process, an estimation of blood oxygen saturation is obtained in real-time. Indeed, the whole process requires only a couple of seconds to obtain the $\mathrm{SpO}_{2}$ value. The methodology was implemented as a web-based application, hence after acquiring the short video, all the processing steps run on a server, and web-services are used for communication. Being web-based, our solution can be employed on different mobile devices (smartphone, tablet, laptop, mobile robots,....), as long as they are equipped with a camera and are able to connect to internet.

The described pipeline was implemented as a prototype in Casalino et al. (2020). Preliminary results under controlled conditions of in-vitro experiments have shown that the methodology is reliable and comparable to a classical pulse 
Fig. 2 Examples of video frames from the PURE Dataset with different setup: steady (a), talking (b), slow translation (c), fast translation $(\mathbf{d})$, small rotation (e) and medium rotation (f)

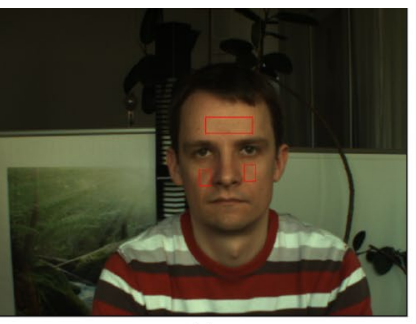

(a)

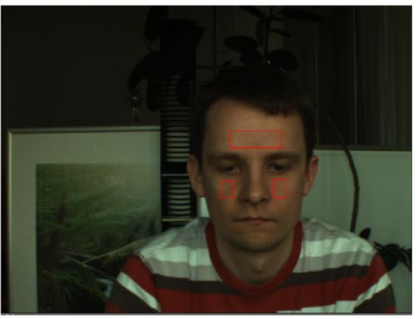

(d)

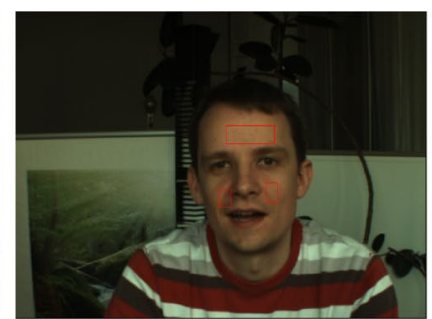

(b)

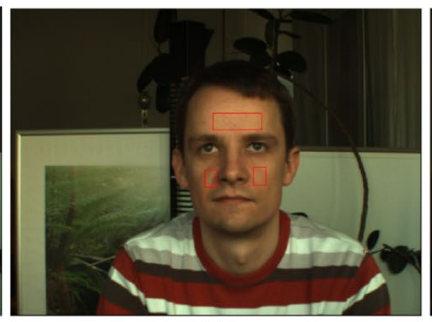

(e)

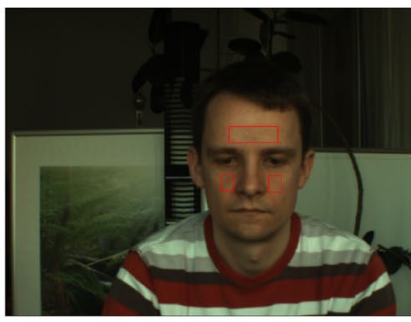

(c)

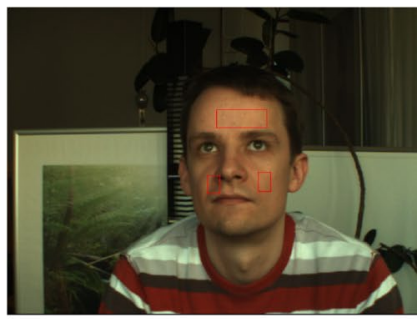

(f) oximeter under controlled conditions, i.e. when the users were required to stay still in front of the camera. However, to definitively assert the robustness of the method, we need to test it in more real scenarios. Actually, in real situations of use of our method on mobile devices, it is essential to determine if the measurement is susceptible to changes in case of head movements. The aim of this work is indeed to evaluate how robust the developed method is to the movements that the user may naturally make during the interaction with the mobile device. To this aim, we have conducted a pilot study to test the method on a dataset including facial videos of users characterized by different head movements. The following section shows the results of this study.

\section{Experiments}

The goal of this work is to examine the influence of artifacts introduced by head motion on the $\mathrm{SpO}_{2}$ measurements made by our contact-less solution originally proposed in Casalino et al. (2020). To this aim we used the PURE dataset (Stricker et al. 2014). It is composed of videos of 10 subjects ( 8 males, 2 females) standing in front of a camera at a distance of about 1.1 meters and performing 6 different head motions, for a total of 60 videos. The videos were recorded with an RGB digital camera. Each video has a length of about 1 minute and was captured at a frame rate of $30 \mathrm{~Hz}$ with a cropped of resolution of $640 \times 480$ pixels. The dataset provides Ground Truth values of heart rate and $\mathrm{SpO}_{2}$ that were measured with a pulse oximeter at the same time as the videos were recorded. The adopted pulse oximeter is a CMS50E with a sampling rate of $60 \mathrm{~Hz}$. Natural lighting condition were used with frontal daylight on the subjects, and changing illumination conditions due to clouds. In all controlled setups the user was sitting in front of the camera. The following six different configurations are considered:

- Steady (S). This setup is used as baseline for measurements. The subject looked at the camera while avoiding head motion.

- Talking (T). The subject talked in front of the camera while avoiding head motion.

- Slow Translation (ST). The subject performed slow head movements parallel to the camera plane.

- Fast Translation (FT). The subject performed head movements parallel to the camera plane, twice faster than in the ST setup.

- Small Rotation (SR). The subject performed a predefined sequence of head rotation (angles about $20^{\circ}$ ).

- Medium Rotation (MR). The subject performed a predefined sequence of head rotation (angles about $35^{\circ}$ ).

Figure 2 shows frames of a single subject in the six different poses. ${ }^{4}$ We processed each video using our method by extracting a number of measurements that is proportional to the length of the video. We obtained an average number of 20 measures for each video. Due to the higher sampling rate of the pulse oximeter, there are more ground truth values than the values obtained by our method. To enable comparison between our measurements and the pulse oximeter measurements, for each video we calculated the mean value of the $\mathrm{SpO}_{2}$ values computed by our system and the mean value of the corresponding $\mathrm{SpO}_{2}$ ground truth values. We quantitatively evaluated the accordance between the

\footnotetext{
${ }^{4}$ Since the dataset is proprietary, we were allowed to show a limited subset of images that are not well representative of the different setups.
} 


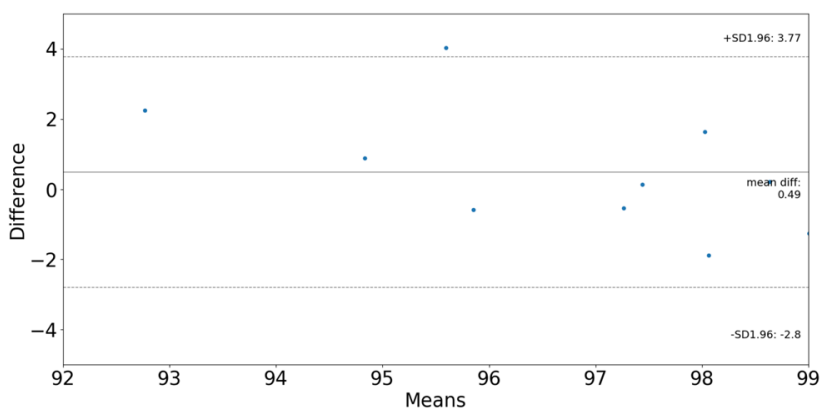

(a)

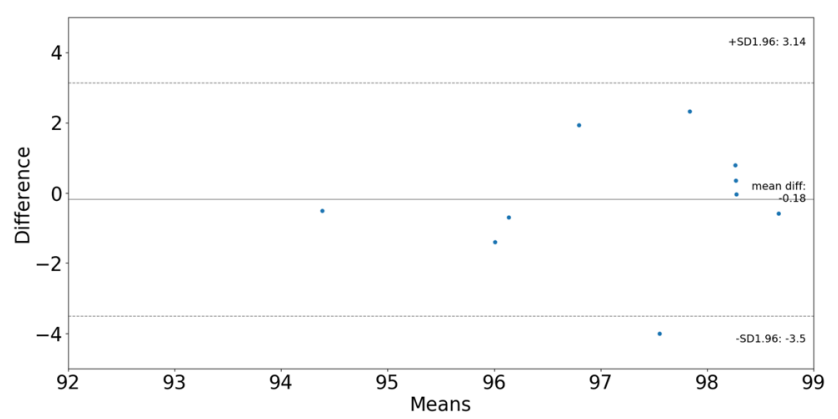

(c)

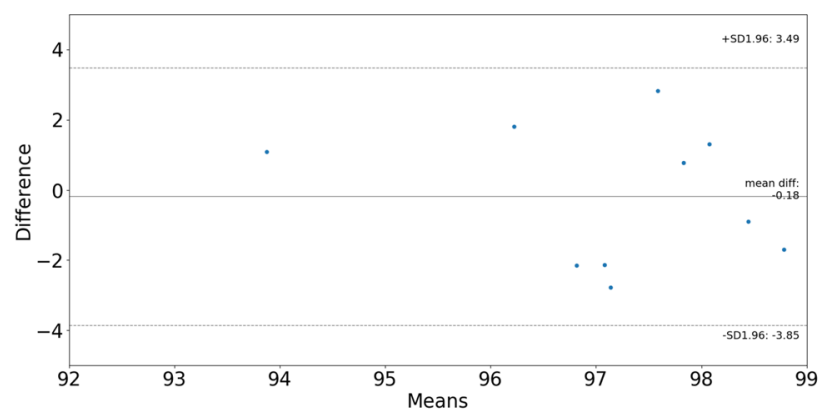

(e)

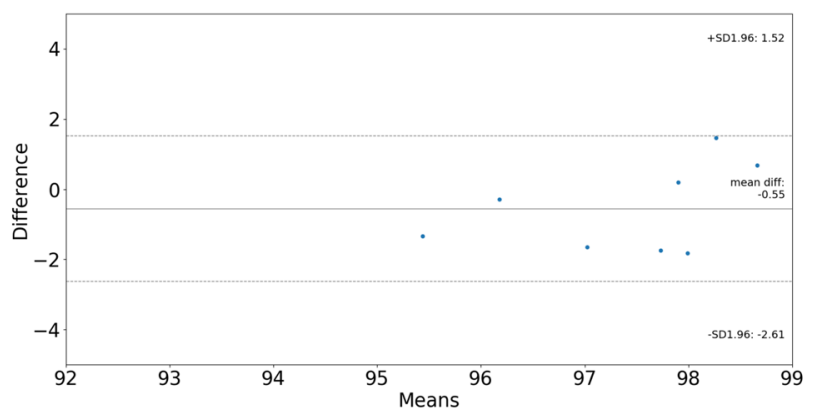

(b)

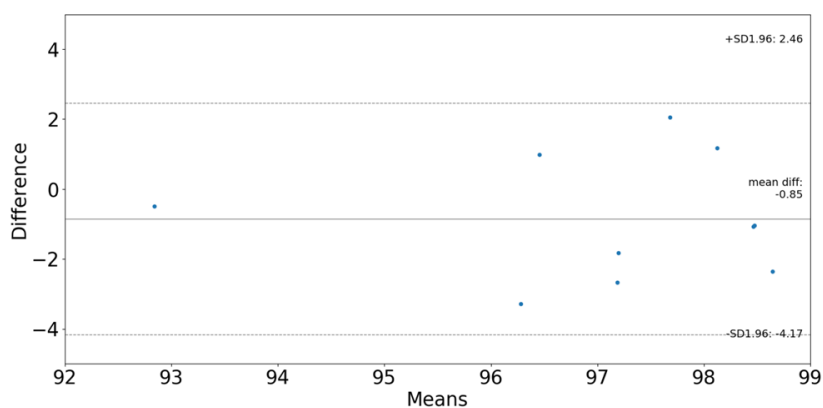

(d)

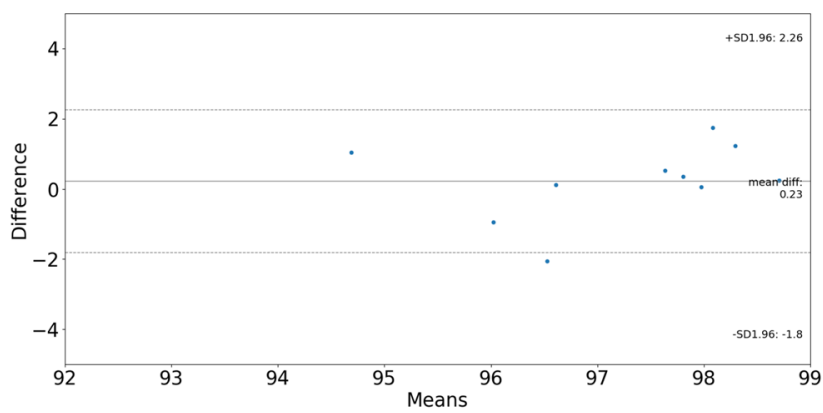

(f)

Fig. 3 Bland-Altman plots of measurements obtained in steady (a), talking (b) slow translation (c), fast translation (d), small rotation (e) and medium rotation (f) setup

two measurements by using Bland-Altman (B\&A) analysis, Pearson Correlation, Mean Absolute Error (MAE), and Root Mean Square Error (RMSE). Figure 5 shows BlandAltman graphs that visualise the differences between the two measurements and define the Level of Agreement (LoA). For reliable measurements the $95 \%$ of the differences should be within the LoA, that are the dotted lines in the graph (lower and upper limits LoA). The central line represents the mean bias. Values lower than zero indicates that the proposed approach under-estimate the ground truth. On the contrary, values higher than zero suggest that the proposed approach returns values that are higher than the real ones. For a good agreement mean bias should be zero or close to it. We highlight that due to measurements errors related to the pulse oximeter, $\mathrm{SpO}_{2}$ is considered valid if the mean bias is within $\pm 2 \%$ (Chan et al. 2019). In detail, Fig. 5 shows the B\&A graph with all mean values of the measures without considering the movement of the subjects. We can observe that there is agreement between the two measurements since all the points except two are within the LoA. In addition, we computed the B\&A graph based on subject motion (Fig. 3). It can be seen that the movements slightly affect the agreement but there are no significant differences among the graphs of different movements. All the points are between the LoA, except for one measurement in settings $S$, ST and MR, that are still very close to the LoA bands. Figure 6 shows the average $\mathrm{SpO}_{2}$ values obtained from ground truth and our system for each subject without accounting for motion. Overall, it can be seen that the values in the plot are in agreement with each other and there are no significant 


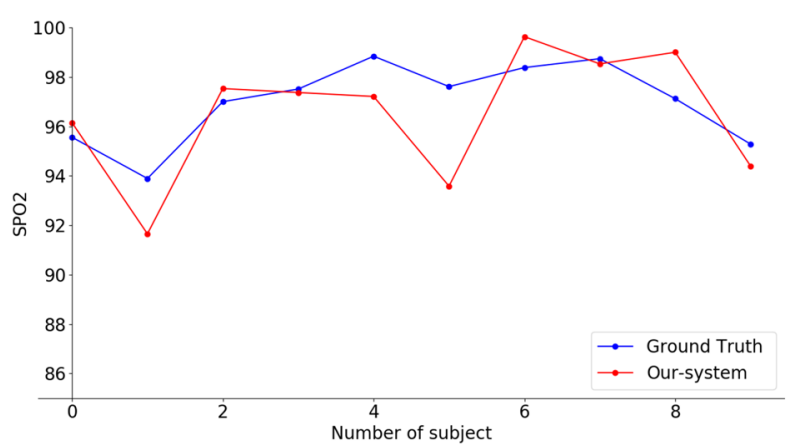

(a)

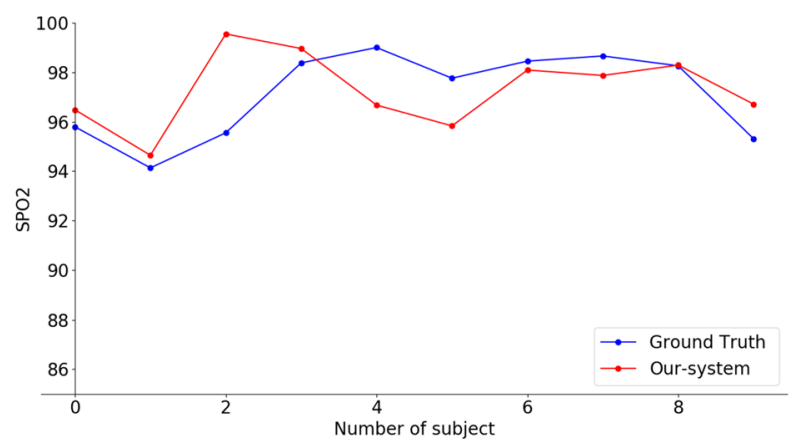

(c)

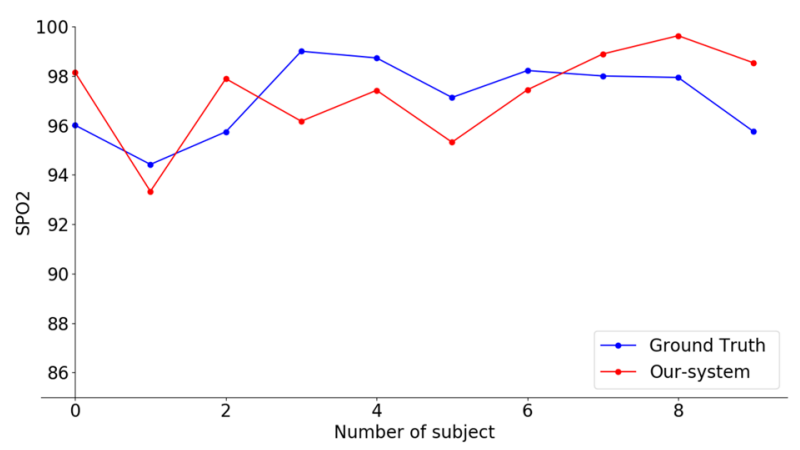

(e)

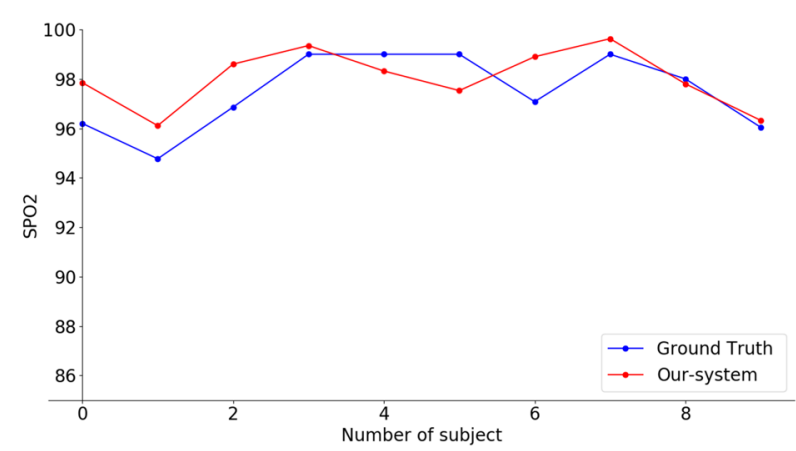

(b)

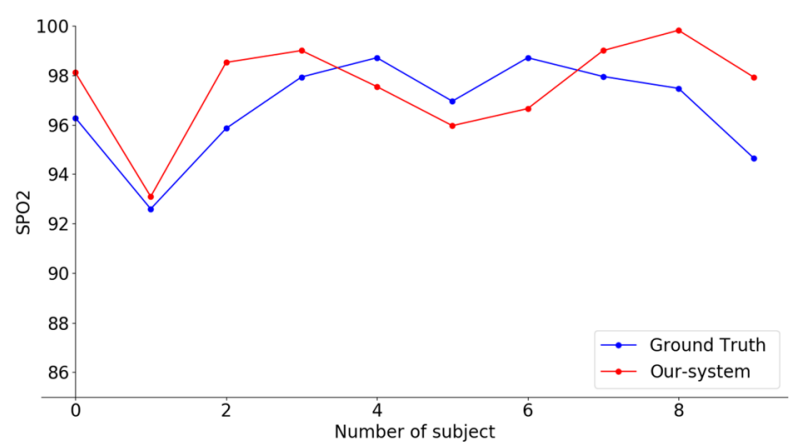

(d)

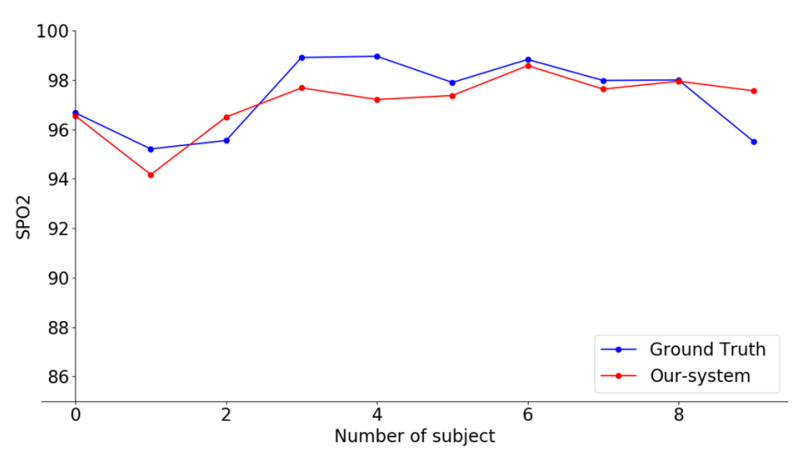

(f)

Fig. 4 Bland-Altman plots of measurements obtained in steady (a), talking (b) slow translation (c), fast translation (d), small rotation (e) and medium rotation (f) setup

differences between the ground truth values and the values in our system. This is also supported by the total MAE value (1.332) in Table 1. In addition, Fig. 4 shows the average values of ground truth and our system, for each subject, in relation to motion. Again, there are no significant differences between the values. Table 1 shows that comparable results of MAE and RMSE are obtained for the six movements. Indeed if we consider the steady case as a baseline, low differences are observed in the other cases. Even with abrupt changes, as fast translation and medium rotation, we obtain low error values. Pearson analysis returns comparable results for the setups S, T, FT and MR. Surprisingly the worst results are obtained with ST and SR setups. We observed that these results depend on significant errors in rPPG acquisitions that were due to bad light conditions. In particular, three out ten subject videos had low lights in the Slow Translations setting. On the contrary, in the Fast Translation setting the scene was well lite, and consequently lower errors were observed. Thus we conclude that low illumination may affect the rPPG-based measurement. However, it is worth noting 
Table 1 Comparison between pulse oximeter and the proposed system

\begin{tabular}{llll}
\hline & Pearson & MAE & RMSE \\
\hline Total & 0.535 & 1.332 & 1.637 \\
Steady & 0.700 & 1.439 & 1.879 \\
Talking & 0.692 & 1.015 & 1.188 \\
Slow translation & 0.407 & 1.262 & 1.701 \\
Fast translation & 0.579 & 1.697 & 1.893 \\
Small rotation & 0.341 & 1.747 & 1.881 \\
Medium rotation & 0.691 & 0.834 & 1.063 \\
\hline
\end{tabular}

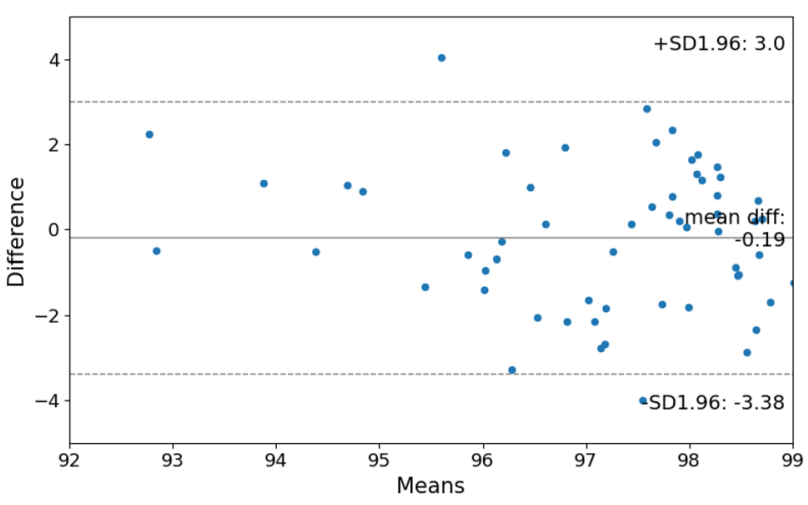

Fig. 5 Bland-Altman plot

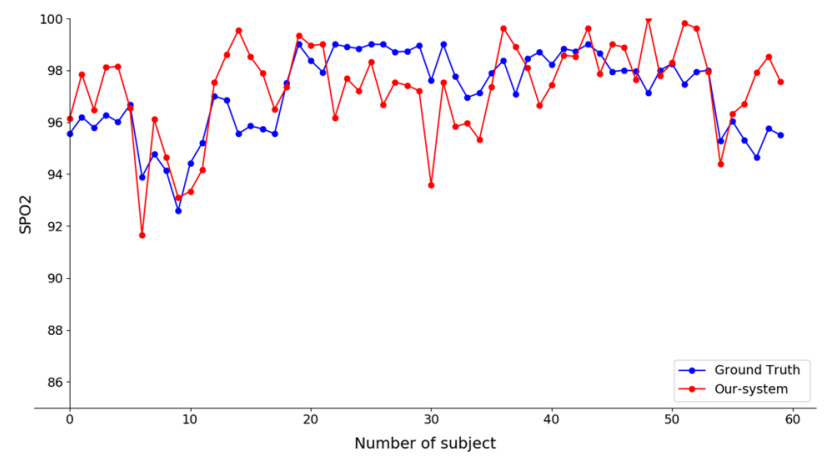

Fig. 6 Comparing the $\mathrm{SpO}_{2}$ values measured by our system with the ground truth

that the proposed methodology acquires the rPPG signal from a sequence of frames that together compose the whole movement. Thus, during real-time acquisition in complex setups such as ST or FT, the user will still be stationary in some frames, as in Steady setup. In fact, in Fig. 2, frames corresponding to ST and FT setups show the user's head in front of the camera. Moreover, even though the setups were controlled, the users might perform slightly different movements. Leveraging these results, we can conclude that $\mathrm{SpO}_{2}$ measurements obtained by the proposed contact-less solution approximate quite well measurements given by the pulse oximeter, and the movements do not affect significantly the estimate of $\mathrm{SpO}_{2}$. This is also obtained thanks to the tracking module that allows the system to cope with head movements such as slow rotations. Indeed in case of head movements, the ROIs are still tracked by the system (as shown in Fig. 2), thus the rPPG signal is still correctly extracted from the ROIs.

\section{Conclusion}

In this paper a mHealth solution for personal and remote measurement of blood oxygen saturation has been presented. The key feature of our contact-less solution is the use of a video camera to acquire video frames of the face from which the plethysmographic signal is extracted and finally an estimate of the $\mathrm{SpO}_{2}$ value is derived. The proposed solution allows a continuous personal control of the blood oxygen saturation in any place, avoiding the use of dedicated measuring devices (e.g. saturometers, pulse oximeters), but simply using a mobile device equipped with a camera. Since it is designed to be as portable as possible, our solution can run on different mobile devices of daily use, thanks to a web-based system, thus making the measurement easy, noninvasive and pervasive. The study in this work was mainly aimed to evaluate the robustness of the proposed approach to different motion setups. Experimental results showed no significant differences among the $\mathrm{SpO}_{2}$ measurements in presence of different slight head movements. We believe the robustness of the method is achieved also thanks to the tracking mechanism that has been implemented as a main step of the methodological pipeline. On the overall, we can conclude that the proposed mHealth solution is robust enough to be used in real scenarios where the user should be free to move and not be forced to be still in front of the camera. This enhances the user experience, as well as the acceptability, leading the user needs to trust the technology and feel safe while using it. However, experimental results have shown a certain influence of the illumination conditions on the acquisition. Future works will be addressed to better study light conditions that could affect the real-time measurement in indoor environments. Moreover, the dataset considered in this work mainly encompasses videos of healthy people. We plan to test the robustness of the method on data coming from sick patients, who have critical values of $\mathrm{SpO}_{2}$. Unfortunately such cases are difficult to collect at the moment, due to the pandemic situation, but we plan to collect more data in the future. With the growing use of mobile technology in the health field, we foresee that patients will increasingly use mobile devices to monitor daily their vital parameters or for personal triage assessment before going to the hospital. For this reason, we are currently extending our solution so as to 
enable contact-less measurement of other vital parameters besides $\mathrm{SpO}_{2}$, such as temperature, heart rate, and breathing rate. Also, we are working to integrate it into a telehealth platform so as the acquired data is sent in real time to physicians who can review it from mobile through an app and from desktop through a web dashboard.

Acknowledgements All the authors are members of the GNCSINDAM Gruppo Nazionale per il Calcolo Scientifico of Istituto Nazionale di Alta Matematica, Francesco Severi, P.le Aldo Moro, Roma, Italy. This work was partially supported by INdAM GNCS within the research project "Computational Intelligence methods for Digital Health". The authors G. Casalino and G. Castellano are with the CITEL - Centro Interdipartimentale di Telemedicina, Università degli studi di Bari Aldo Moro.

\section{References}

Allado E, Poussel M, Moussu A, Saunier V, Bernard Y, Albuisson E, Chenuel B (2021) Innovative measurement of routine physiological variables (heart rate, respiratory rate and oxygen saturation) using a remote photoplethysmography imaging system: a prospective comparative trial protocol. BMJ Open. https://doi.org/ 10.1136/bmjopen-2020-047896

Allen J (2007) Photoplethysmography and its application in clinical physiological measurement. Physiol Meas 28(3):R1

Ardito C, Di Noia T, Fasciano C, Lofù D, Macchiarulo N, Mallardi G, Pazienza A, Vitulano F (2020) Management at the edge of situation awareness during patient telemonitoring. International conference of the italian association for artificial intelligence. Springer, Berlin, pp 372-387

Baldinger M, Heinrich A, Adams T, Martens E, Dommasch M, Müller A, Siegmann A, Schmidt G, Amft O, Laerhoven KV (2021) TELECOVID: remote vital signs monitoring of COVID-19 risk patients in home isolation with an in-ear wearable. IEEE Pervasive Comput 20(2):58-62

Casalino G, Castellano G, Zaza G (2020) A mhealth solution for contact-less self-monitoring of blood oxygen saturation. In: 2020 IEEE Symposium on Computers and Communications (ISCC), IEEE, pp 1-7

Casalino G, Castellano G, Kaczmarek-Majer K, Hryniewicz O (2021) Intelligent analysis of data streams about phone calls for bipolar disorder monitoring. In: 2021 IEEE International Conference on Fuzzy Systems (FUZZ-IEEE), IEEE, pp 1-6

Challoner A (1979) Photoelectric plethysmography for estimating cutaneous blood flow. Non-invasive physiological measurements. Academic Press, London, pp 125-151

Chan C, Inskip J, Kirkham A, Ansermino J, Dumont G, Li L, Ho K, Lauscher HN, Ryerson C, Hoens A et al (2019) A smartphone oximeter with a fingertip probe for use during exercise training: usability, validity and reliability in individuals with chronic lung disease and healthy controls. Physiotherapy 105(3):297-306

Dasari A, Prakash SKA, Jeni LA, Tucker CS (2021) Evaluation of biases in remote photoplethysmography methods. NPJ Digit Med $4(1): 1-13$

De Haan G, Jeanne V (2013) Robust pulse rate from chrominancebased rppg. IEEE Trans Biomed Eng 60(10):2878-2886

El-Rashidy N, El-Sappagh S, Islam SMR, El-Bakry HM, Abdelrazek S (2020) End-to-end deep learning framework for coronavirus (covid-19) detection and monitoring. Electronics 9(9):1439

El-Rashidy N, El-Sappagh S, Islam SMR, El-Bakry MH, Abdelrazek S (2021) Mobile health in remote patient monitoring for chronic diseases: principles, trends, and challenges. Diagnostics. https:// doi.org/10.3390/diagnostics11040607

Hassan MA, Malik AS, Fofi D, Saad N, Karasfi B, Ali YS, Meriaudeau $F$ (2017) Heart rate estimation using facial video: a review. Biomed Signal Process Control 38:346-360

Kim AY, Choi WS (2020) Considerations on the implementation of the telemedicine system encountered with stakeholders' resistance in Covid-19 pandemic. Telemed e-Health 27:475-480

Kim JM, Arakawa K, Benson KT, Fox DK (1986) Pulse oximetry and circulatory kinetics associated with pulse volume amplitude measured by photoelectric plethysmography. Anesth Analg 65(12):1333-1339

Kondylakis H, Katehakis DG, Kouroubali A, Logothetidis F, Triantafyllidis A, Kalamaras I, Votis K, Tzovaras D (2020) Covid-19 mobile apps: a systematic review of the literature. J Med Internet Res 22(12):e23170

Kong L, Zhao Y, Dong L, Jian Y, Jin X, Li B, Feng Y, Liu M, Liu X, Wu H (2013) Non-contact detection of oxygen saturation based on visible light imaging device using ambient light. Opt Express 21(15):17464-17471

Liu Y, Qin B, Li R, Li X, Huang A, Liu H, Lv Y, Liu M (2021) Motion-robust multimodal heart rate estimation using bcg fused remote-ppg with deep facial roi tracker and pose constrained Kalman filter. IEEE Trans Instrum Meas 70:1-15

Lupton D (2013) The digitally engaged patient: Self-monitoring and self-care in the digital health era. Social Theory Health 11(3):256-270

Lurie N, Carr BG (2018) The role of telehealth in the medical response to disasters. JAMA Intern Med 178(6):745-746

Moses JC, Adibi S, Shariful Islam SM, Wickramasinghe N, Nguyen L (2021) Application of smartphone technologies in disease monitoring: a systematic review. Healthcare. https://doi.org/10. 3390/healthcare9070889

Naranjo-Rojas A, Perula-de Torres LÁ, Cruz-Mosquera FE, MolinaRecio G (2021) Mobile application for monitoring patients under home oxygen therapy: a protocol for a randomized controlled trial. BMC Fam Pract 22(1):1-10

Poh MZ, McDuff DJ, Picard RW (2010) Non-contact, automated cardiac pulse measurements using video imaging and blind source separation. Opt Express 18(10):10762-10774

Rouast PV, Adam MT, Chiong R, Cornforth D, Lux E (2018) Remote heart rate measurement using low-cost rgb face video: a technical literature review. Front Comp Sci 12(5):858-872

Sabbadini R, Tocco JD, Massaroni C, Schena E, Carassiti M (2021) A smart face mask based on photoplethysmography for cardiorespiratory monitoring in occupational settings. In: IEEE International Symposium on Medical Measurements and Applications, MeMeA 2021, Lausanne, Switzerland, June 23-25, 2021, IEEE, pp 1-6

Sahoo PK, Thakkar HK, Lee M (2017) A cardiac early warning system with multi channel SCG and ECG monitoring for mobile health. Sensors 17(4):711

Solomon O Jr (1991) Psd computations using Welch's method. STIN 92:23584

Song R, Li J, Wang M, Cheng J, Li C, Chen X (2021) Remote photoplethysmography with an eemd-mcca method robust against spatially uneven illuminations. IEEE Sens J. https://doi.org/10. 1109/JSEN.2021.3067770

Speake T, Mersereau R (1981) A note on the use of windows for twodimensional fir filter design. IEEE Trans Acoust Speech Signal Process 29(1): 125-127

Speth J, Vance N, Flynn P, Bowyer K, Czajka A (2021) Remote pulse estimation in the presence of face masks. arXiv preprint arXiv: 210104096

Storz P, Wickner S, Batt B, Schuh J, Junger D, Möller Y, Malek N, Thies C (2021) bwhealthapp: A software system to support 
personalized medicine by individual monitoring of vital parameters of outpatients. In: HEALTHINF, pp 613-620

Stricker R, Müller S, Groß H (2014) Non-contact video-based pulse rate measurement on a mobile service robot. In: The 23rd IEEE International Symposium on Robot and Human Interactive Communication, pp 1056-1062

Swan M (2012) Health 2050: the realization of personalized medicine through crowdsourcing, the quantified self, and the participatory biocitizen. J Personal Med 2(3):93-118

Tayfur I, Afacan MA (2019) Reliability of smartphone measurements of vital parameters: a prospective study using a reference method. Am J Emerg Med 37:1527-1530

Tomlinson S, Behrmann S, Cranford J, Louie M, Hashikawa A (2018) Accuracy of smartphone-based pulse oximetry compared with hospital-grade pulse oximetry in healthy children. Telemed e-Health 24(7):527-535

Tripathi G, Abdul Ahad M, Paiva S (2020) Sms: a secure healthcare model for smart cities. Electronics 9(7):1135
Verkruysse W, Svaasand LO, Nelson JS (2008) Remote plethysmographic imaging using ambient light. Opt Express 16(26):21434-21445

Villarroel M, Jorge J, Meredith D, Sutherland S, Pugh C, Tarassenko L (2020) Non-contact vital-sign monitoring of patients undergoing haemodialysis treatment. Sci Rep 10(1):1-21

Yang Z, Wang H, Lu F (2021) Assessment of deep learning-based heart rate estimation using remote photoplethysmography under different illuminations. arXiv preprint arXiv:210713193

Publisher's Note Springer Nature remains neutral with regard to jurisdictional claims in published maps and institutional affiliations. 